\title{
Extraction Process Validation of Isatis Radix
}

\author{
Junshan Li, Bo Zhang \\ Shineway Pharmaceutical Group Ltd., Shijiazhuang, China \\ Email: swljs@sina.com
}

Received 20 January 2014; revised 8 March 2014; accepted 26 March 2014

Copyright (C) 2014 by authors and Scientific Research Publishing Inc.

This work is licensed under the Creative Commons Attribution International License (CC BY).

http://creativecommons.org/licenses/by/4.0/

(c) (i) Open Access

\begin{abstract}
This study is aimed to validate the extraction process of Isatis Radix. Using near infrared spectroscopy and high performance liquid chromatography to determine the uridine, adenosine, guanosine contents in Isatis Radix Solution, as well as NIR to monitor the extraction process. The results indicate that the existing extraction is optimized to extract thoroughly.
\end{abstract}

\section{Keywords}

Isatis Radix; Extraction Process; NIR Spectroscopy; HPLC; Validation

\section{Introduction}

Isatis Radix is the dried root of cruciferous plant Isatis indigotica Fort. It is traditionally used to clear away heat \& detoxify, cool the blood and benefit the throat [1]. And it is a medicine for epidemic prevention and slight cold. The traditional extraction process is decocting the Isatis Radix ingredients with 6 - 10 times of water two times, and each time is about half an hour. The modern extraction process is the first extraction that added 8 times of water, and kept temperature at $98^{\circ} \mathrm{C}-100^{\circ} \mathrm{C}$ for 1 hour; and the second extraction added 6 times of water, and kept temperature at $98^{\circ} \mathrm{C}-100^{\circ} \mathrm{C}$ for 0.5 hour. As the Isatis Radix alkaloids (uridine, adenosine, Guanosine, etc.) having significant antiviral activity [2], this study uses near infrared spectroscopy and high performance liquid chromatography technology to detect uridine, adenosine and guanosine in Isatis Radix extraction solution, and to verify the modern industrial production of Isatis Radix.

\section{Methods}

\subsection{Equipments and Materials}

Model 7020 multifunction extracting tank (Wuhan Pharmaceutical Machinery Factory); near infrared spectrometer (Focused Photonics Inc.); Diane U3000-HPLC (with diode array detector and chameleon chromatography workstation); Column: Phenomenexluna RP C18, $4.6 \times 250 \mathrm{~mm}, 5 \mu \mathrm{m}$. 
Isatis Radix is accredited by Chinese Medicine Expert Sun Baohui to be the dried roots of cruciferous plant Isatis indigotica (Batch number: 120605, 120610, 120612, 120624, 120702, 120708, 120715, 120726, 120812, 120825, 120827, 120831, 120915, 120918, 120923, 120928, 121010, 121016, 121020, 121025). Uridine (Batch number: 110887 - 200202) and Adenosine (Batch number: 110879 - 200202). Reference Substances are purchased from National Institutes for Food and Drug Control. Guanosine (CAS NO: 118-00-3). Reference Substances are purchased from Simga Co. Acetonitrile is chromatographic pure, while water is purified water and the other reagents are analytical pure.

\subsection{Production of Isatis Radix Extraction}

Wash the Isatis Radix, cut $200 \mathrm{~kg}$ into pieces, and put them into multi-functional tank. Add water with 8 times of Isatis Radix for the first extraction, and keep temperature at $100^{\circ} \mathrm{C}$ for 1 hour. Add water with 6 times of Isatis Radix for the second extraction, and keep temperature at $100^{\circ} \mathrm{C}$ for 0.5 hour. Mix the two times extraction to be the extraction solution.

\subsection{Sample}

Take samples in the circulation pipeline at the side of extraction tank during producing. Samples flow through NIR channel and then loop back to the extraction tank. NIR channel is always flowing with extraction solution from the starting to the ending. NIR carry out a full scan analysis every two minutes. Take the first nine batches samples out to be the extraction reference solution and analyze them.

\subsection{Spectrum Condition}

NIR scanning mode: optic fiber transmission scan at spectral range $1000-2500 \mathrm{~nm}$, and resolution is $1 \mathrm{~nm}$. Use the air as the blank, and scan 16 times with double benefit (Figure 1).

\subsection{Chromatographic Conditions}

\subsubsection{Chromatographic System Suitability Test}

Use the $0.1 \%$ formic acid solution as mobile phase $\mathrm{A}$, and acetonitrile as mobile phase $\mathrm{B}$. Gradient elute according to Table 1 gradient (gradient elution); flow rate is $0.5 \mathrm{ml} / \mathrm{min}$, and column temperature is $25^{\circ} \mathrm{C}$; detection wavelength is $254 \mathrm{~nm}$. Inject $10 \mu \mathrm{l}$ test solution accurately to HPLC, determine, and record 6 minutes chromatography then quantified by using external standard method.

\subsubsection{Preparation of the Reference Solution}

Accurately weigh uridine, adenosine, Guridine Reference Substances to the same flask and prepare the solution at $0.1 \mathrm{mg} / \mathrm{ml}$ each of the three components (Figures 2 and 3).

\subsubsection{Methodology Validation}

Take the extraction solution made from batch 120605 Radix extract to test the precision, linear, repeatability and recovery of above chromatographic conditions. All are in good results as indicated in Table 2.

\subsubsection{Sample Detection}

Use the above chromatographic conditions to detect the 9 batches of extract solution. The content of Uridine, Adenosine and Guridine are calculated by external standard method, and the content scope is in Table 3. Use

Table 1. Gradient elution spectrum conditions of Isatis Radix.

\begin{tabular}{ccc}
\hline Time (min) & B (\%) & A (\%) \\
$0-42$ & $0 \rightarrow 12$ & $100 \rightarrow 88$ \\
$42-65$ & $12 \rightarrow 19$ & $88 \rightarrow 81$ \\
$65-75$ & $19 \rightarrow 100$ & $8 \rightarrow 10$ \\
$75-85$ & $100 \rightarrow 0$ & $0-100$ \\
\hline
\end{tabular}


Table 2. Chromatographic conditions methodology validation study.

\begin{tabular}{ccccccc}
\hline Components & Precision/\% & Linear Equation & Correlation Coefficients Repeatability/\% & Recovery Result/\% & Recovery RSD/\% \\
\hline Uridine & 0.21 & $\mathrm{y}=31.81 \mathrm{x}+133.65$ & 0.9997 & 0.78 & 98.34 & 0.89 \\
Adenosine & 0.25 & $\mathrm{y}=45.85 \mathrm{x}+266.18$ & 0.9992 & 0.62 & 98.23 & 0.96 \\
Guridine & 0.15 & $\mathrm{y}=34.40 \mathrm{x}+242.89$ & 0.9994 & 0.39 & 98.16 & 0.37 \\
\hline
\end{tabular}

Table 3. Isatis radix contrast model parameters.

\begin{tabular}{|c|c|c|c|c|c|c|c|c|}
\hline Ingr. & $\begin{array}{l}\text { Extr. } \\
\text { Time }\end{array}$ & $\begin{array}{c}\text { Content } \\
\mu \mathrm{g} / \mathrm{ml}\end{array}$ & $\begin{array}{c}\text { Spectra } \\
\text { Treatment }\end{array}$ & $\begin{array}{l}\text { Spectrum } \\
\text { Scope }\end{array}$ & $\begin{array}{c}\text { Main } \\
\text { Factors }\end{array}$ & $\begin{array}{l}\text { Correlation } \\
\text { Coefficient }\end{array}$ & $\begin{array}{c}\text { Calibrated } \\
\text { Std Dev }\end{array}$ & RMSEP \\
\hline \multirow{2}{*}{ Rridine } & $1^{\text {st }}$ extr. & $34-68$ & Original & 1312 - 1845 nm & 6 & 0.992 & 0.247 & 0.452 \\
\hline & $2^{\text {nd }}$ extr. & $13-23$ & Original & 1312 - 1845 nm & 4 & 0.995 & 0.0642 & 0.0826 \\
\hline \multirow{2}{*}{ Adenosine } & $1^{\text {st }}$ extr. & $40-87$ & Original & $1354-1840 \mathrm{~nm}$ & 7 & 0.997 & 0.386 & 0.536 \\
\hline & $2^{\text {nd }}$ extr. & $19-31$ & Original & $1354-1840 \mathrm{~nm}$ & 4 & 0.994 & 0.0587 & 0.0692 \\
\hline \multirow{2}{*}{ Guanosine } & $1^{\text {st }}$ extr. & $29-63$ & Original & $1287-1840 \mathrm{~nm}$ & 5 & 0.998 & 0.324 & 0.478 \\
\hline & $2^{\text {nd }}$ extr. & $12-20$ & Original & $1287-1840 \mathrm{~nm}$ & 6 & 0.995 & 0.0542 & 0.0783 \\
\hline
\end{tabular}

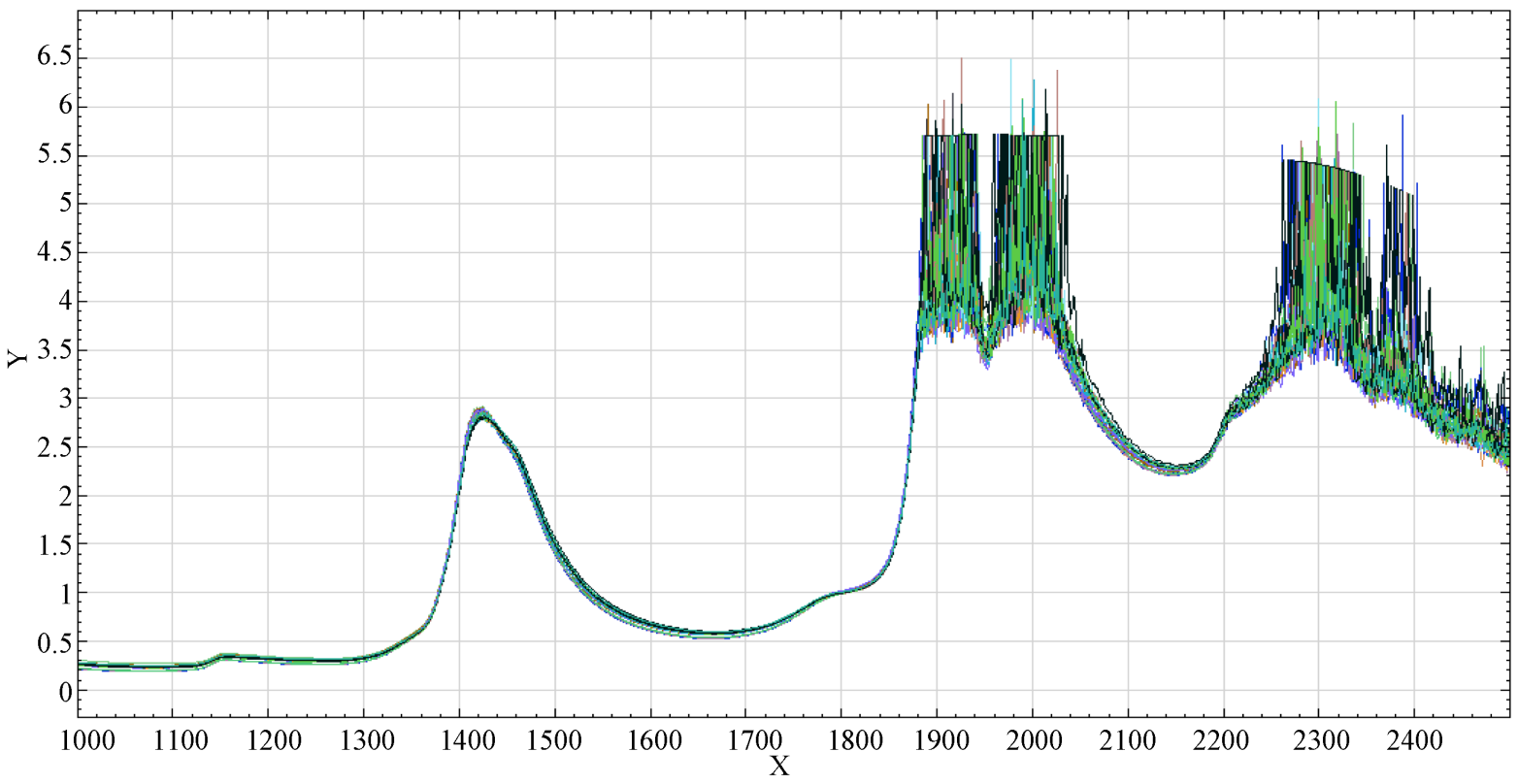

Figure 1. NIR spectrum of isatis radix.

the extraction time as abscissa and the content of each component as a vertical coordinate. Each of contents showed the map as Figures 4-6. Comparison the content changing trends of 9 batches of sample extraction. The variation trends are similar.

\subsection{NIR Prediction Model}

Select 10 spectrums of each batch from first extractions in total 9 batch (take 20 for first batch) while 10 spectrums from second extractions of each batch (take 15 for first and second batches). Establish Content Prediction Model of each extraction in line with the corresponding NIR and predict the contents of above mentioned 3 components. Parameters of sub-models to each component are shown in Table 3. Predict the contents of re- 


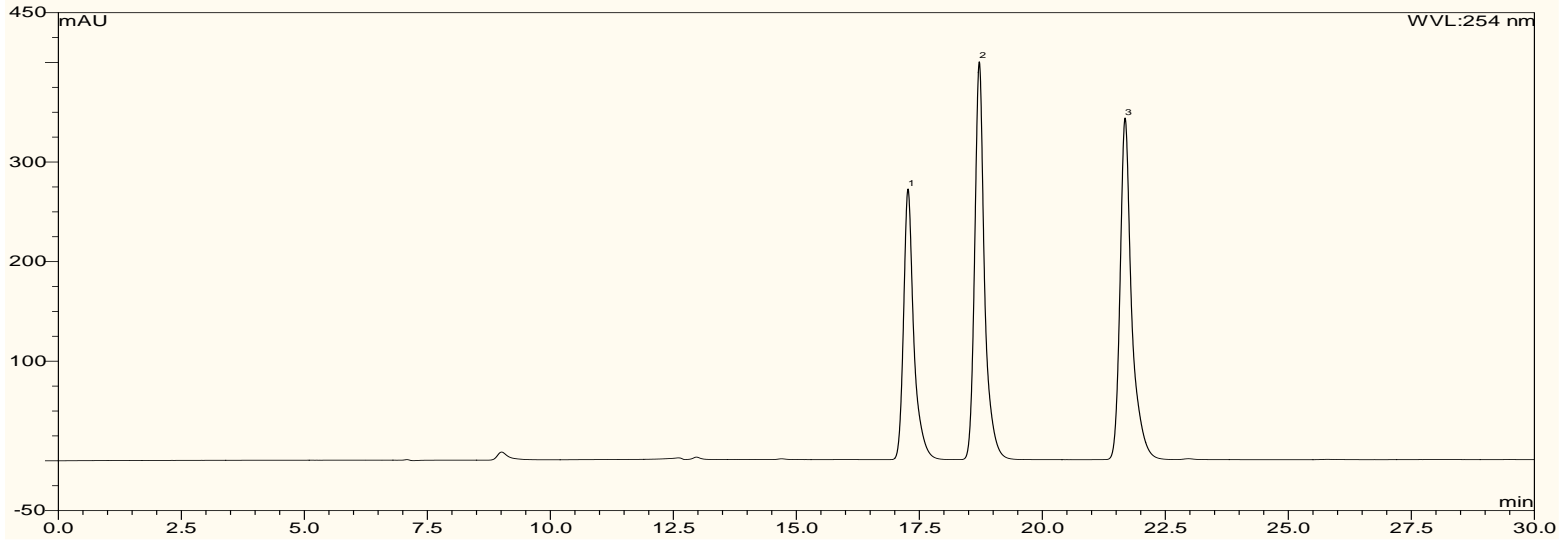

Figure 2. Spectrum of mixed RS of Isatis Radix.

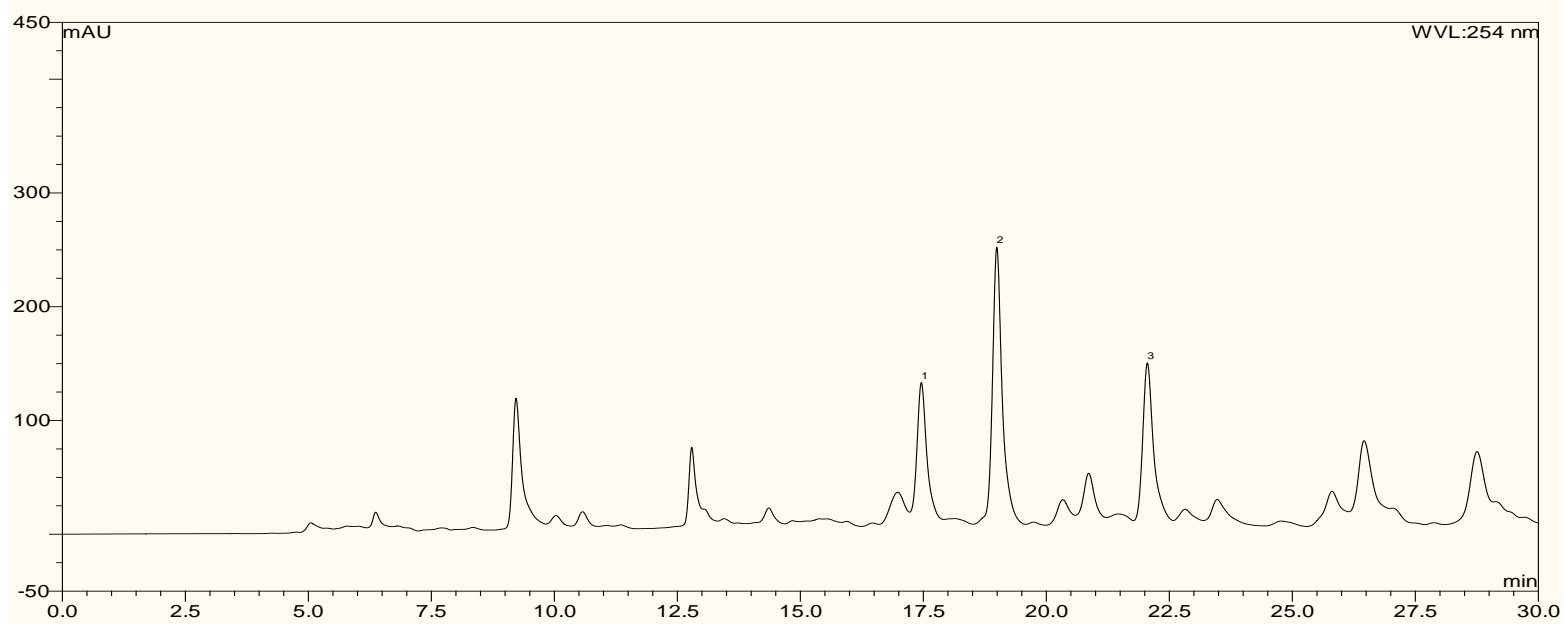

Figure 3. Spectrum of Isatis Radix sample.

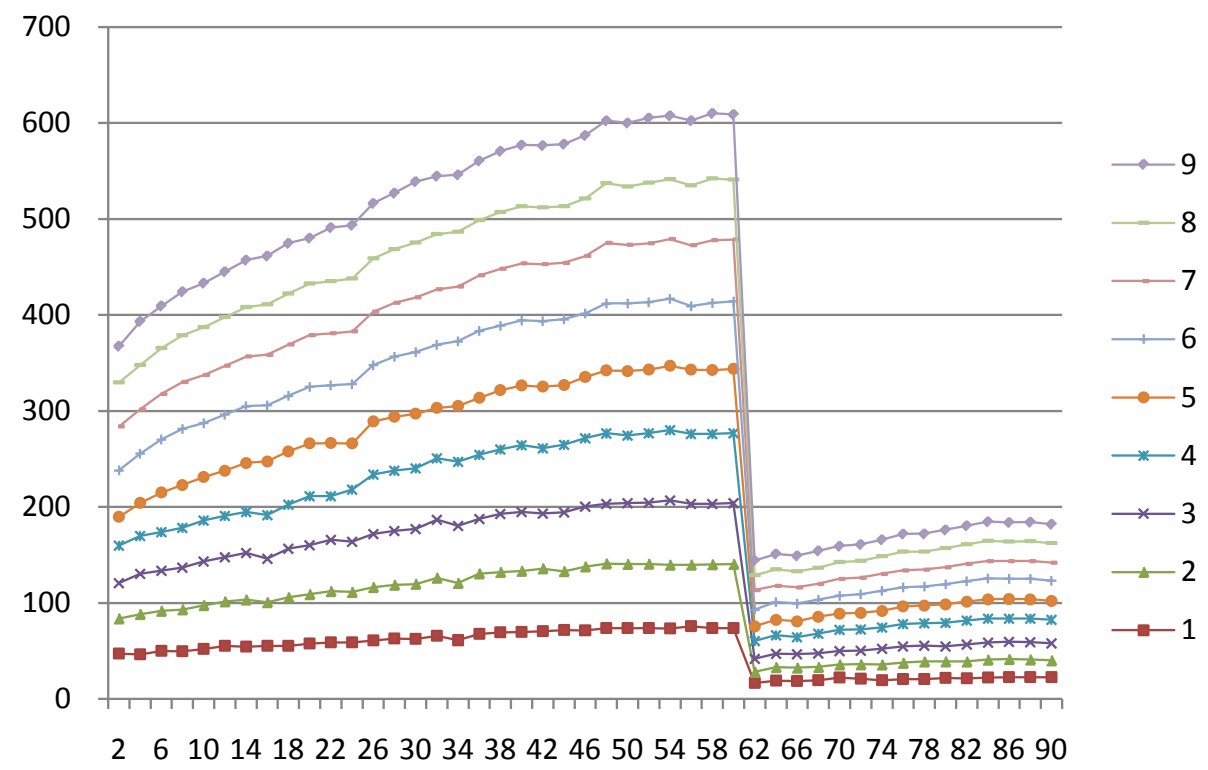

Figure 4. Figure of uridine trend by HPLC. 


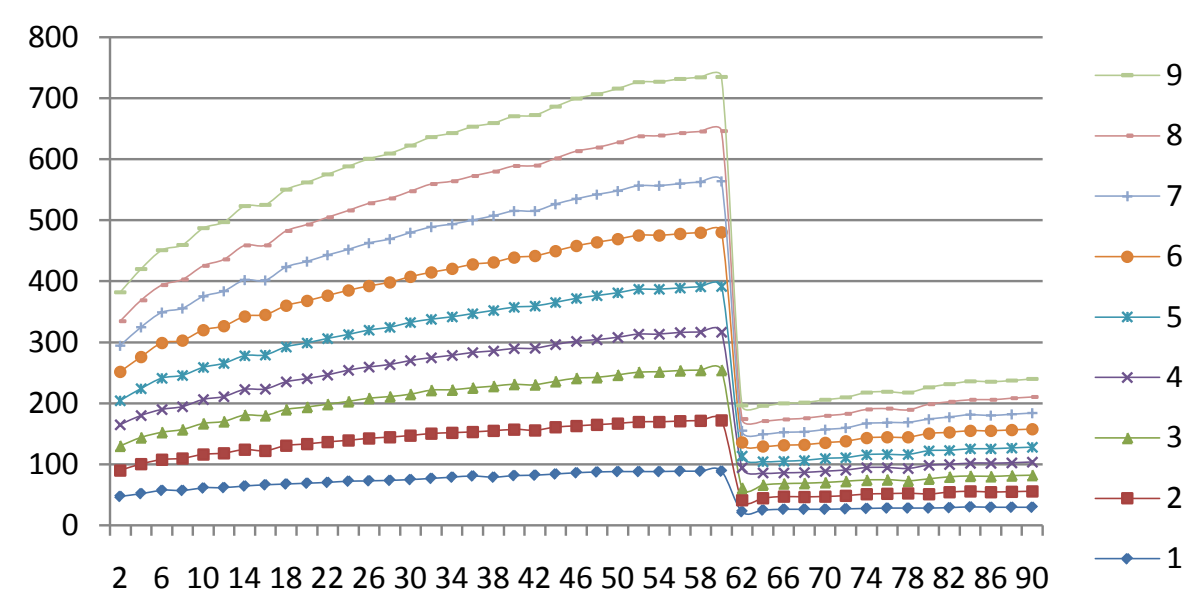

Figure 5. Figure of adenosine trend by HPLC.

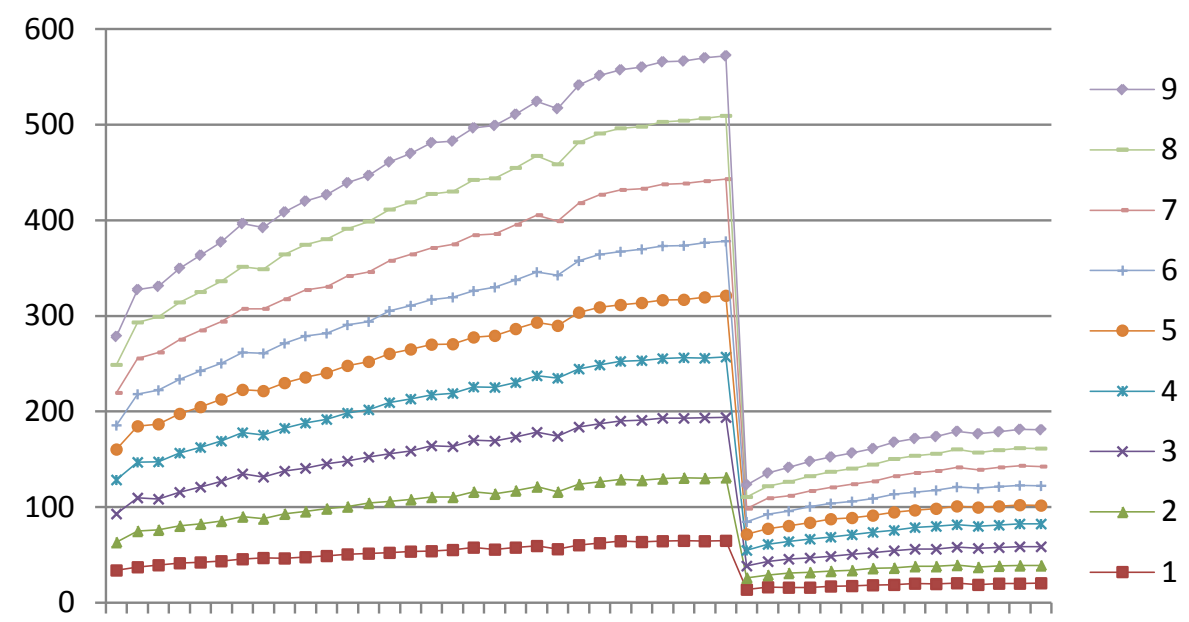

26101418222630343842465054586266707478828690

Figure 6. Figure of guanosine trend by HPLC.

maining samples except the ones used for model establishment in nine batches; compare the contents with the results obtained by HPLC. And relative deviations of the results vary from $0.2 \%$ to $3.7 \%$ which mean good correlation (Figure 7).

\subsection{Results and Analysis}

See Figure 8 to find the NIR prediction trend of each component of contents on Batch 120910 Isatis Radix. In first extraction, all component s gradually increase and reach equilibrium at around 50 minutes. In second extraction, the trend of each active component show solwly increase and reach equilibrium at around 20 minutes, and then trends of the content exponential of all components are gradually lower \& slow. Contents of each component in 20 batches of Isatis Radix extraction solution have tiny deviation at the same time interval, as well as a similar exponential trend line which are gradually lowered and slowed in Figures 9-11.

\section{Discussion}

1) Different batches of Isatis Radix extraction solution under the current production procedures share a similar content as well as the variation trend which means that the extraction method is stable. In the industrial production, the first extraction takes longer time than the second extraction on reaching equilibrium due to the huge input quantities, slow heating and other factors reaching equilibrium. In the first extraction, it takes 50 minutes for uri- 


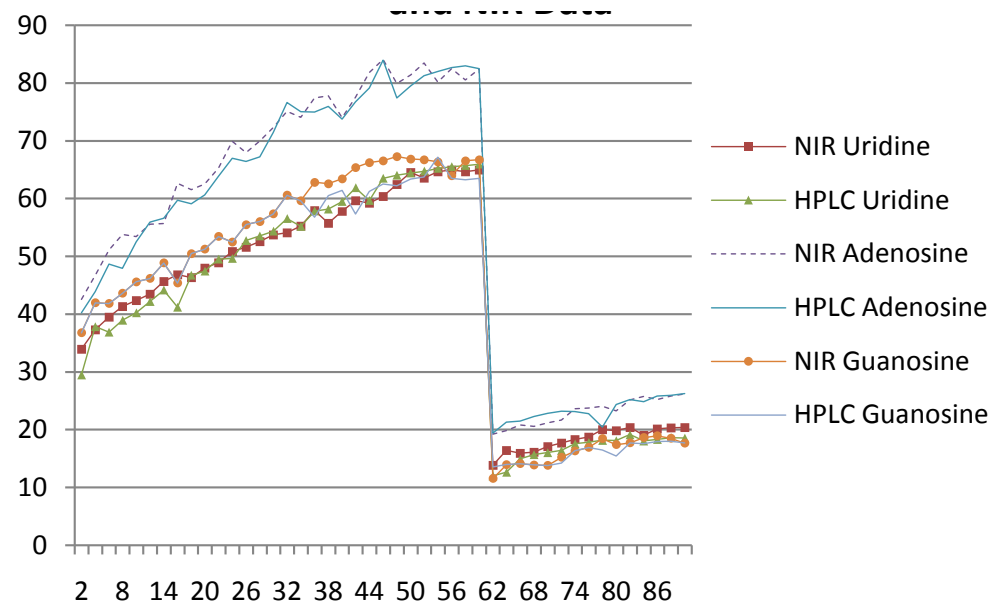

Figure 7. Figure of comparation between HPLC data and NIR data.

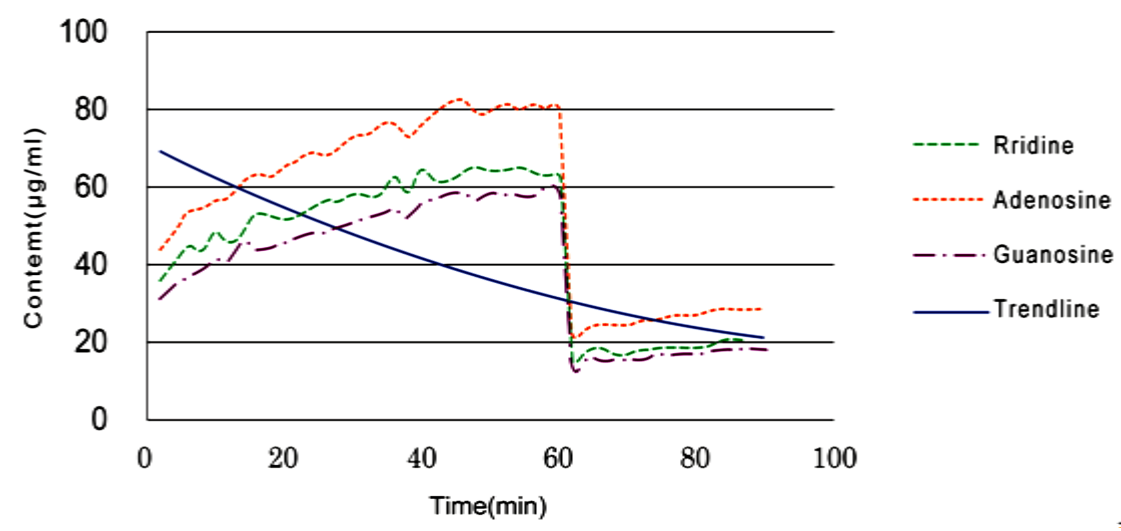

Figure 8. Content trend of each component in Isatis Radix extraction solution.

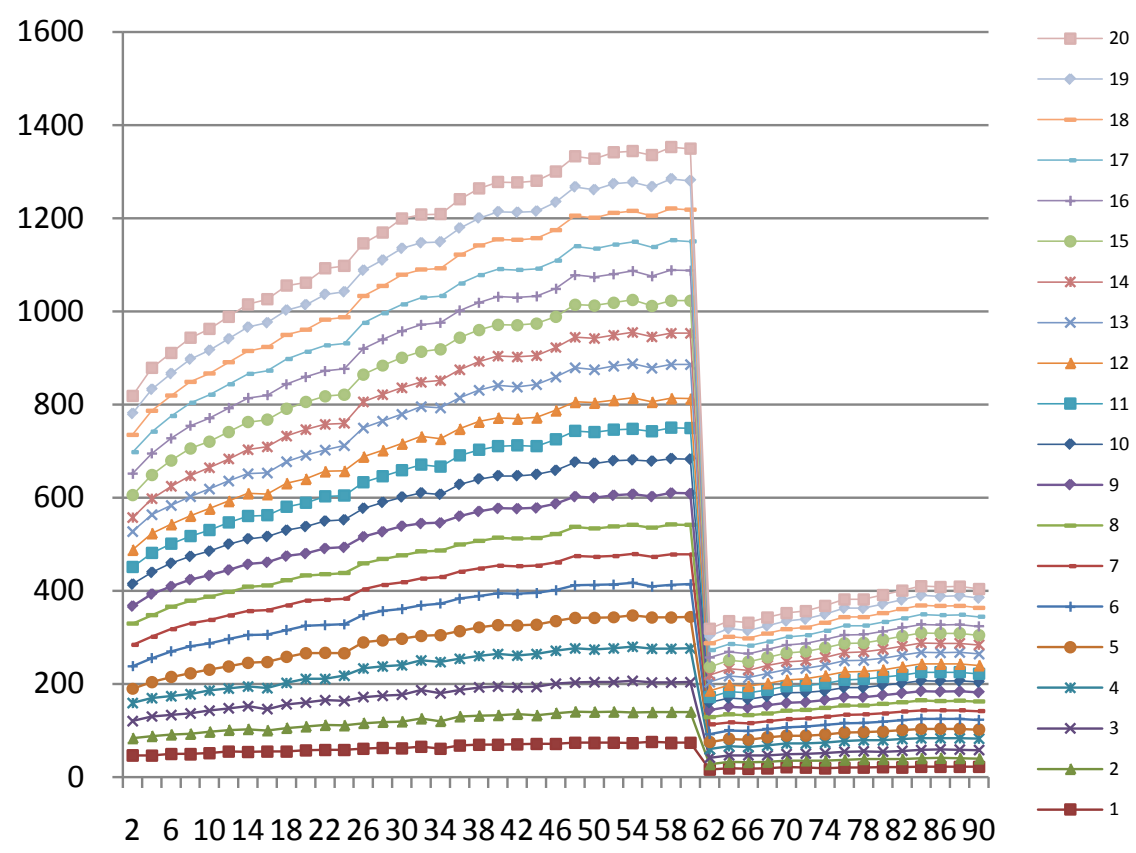

Figure 9. Figure of uridine trend by NIR. 


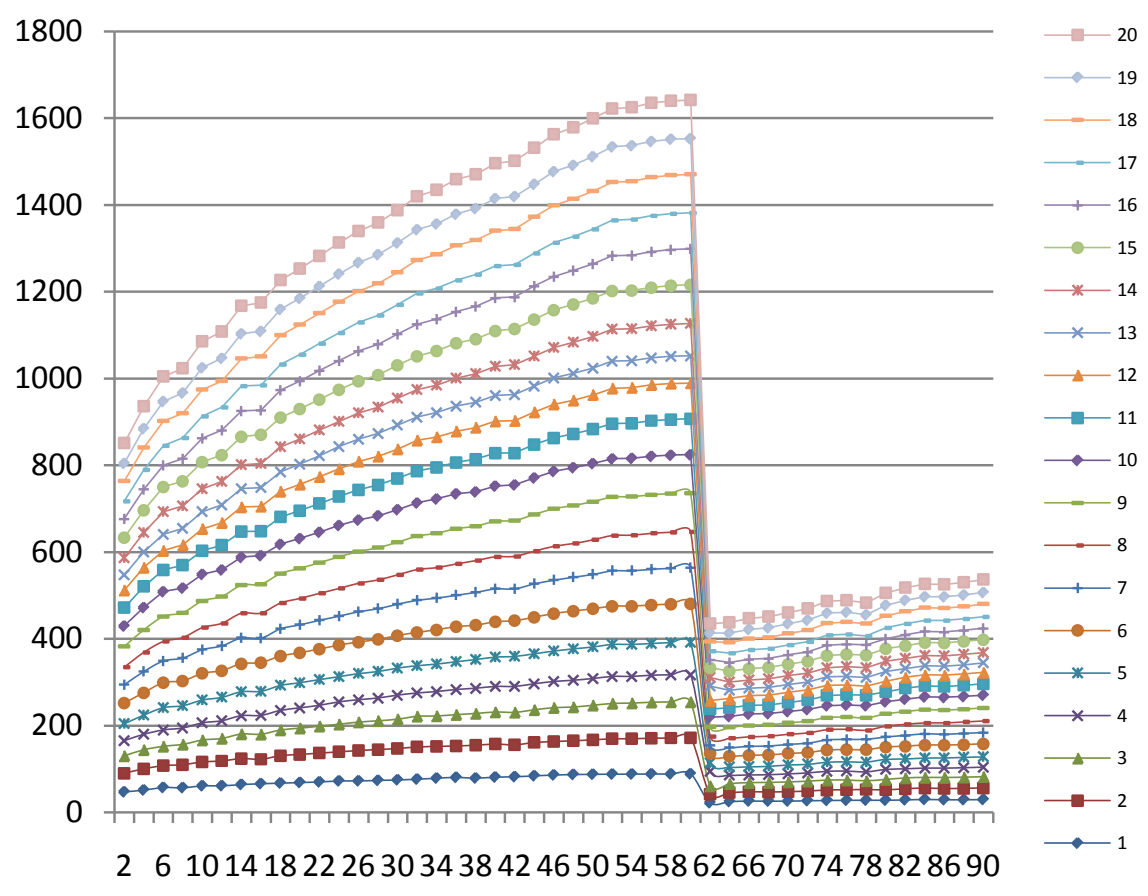

Figure 10. Figure of adenosine trend by NIR.

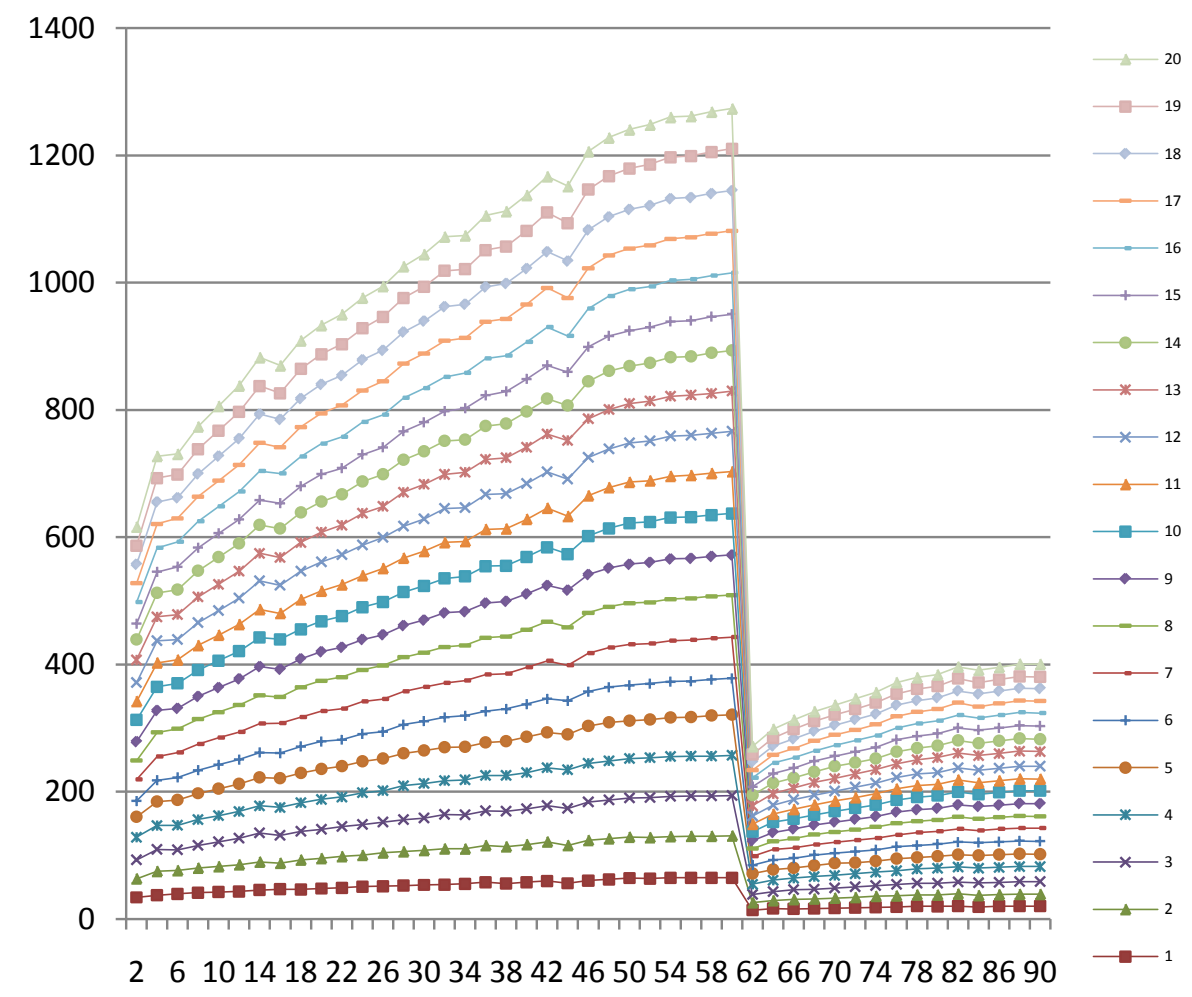

Figure 11. Figure of guanosine trend by NIR.

dine, adenosine, guanosine content to reach equilibrium while 20 minutes for second extraction. When exploring the extraction methods in lab scale, the third extraction obtains less than one-tenth of content compared to the first extraction, so we discard the third extraction according to economic guideline. We can conclude that the 
current Isatis Radix extraction method is the best one.

2) As the long time of analysis and complex operation and complicated calculation of HPLC, the analysis of chromatographic data will take about 2 hours from sampling to take calculated results. And the near infrared method just needs less than 3 minutes. To save time and cost, using the near infrared method is the best choice. Use the first 9 groups to establish near infrared model and near infrared data by chromatographic data validation. When matched, between near infrared detection results and chromatographic detection results of the same data, the deviations are within five percent and most of them within two percent. The monitoring data in industrial production can use near infrared detection instead of HPLC.

3) This study just investigates the content of uridine, adenosine and guanosine of existing Isatis Radix production process. And for other active components such as alkaloids, amino acids, polysaccharides etc. in Isatis Radix, further studies are needed.

4) The Isatis Radix studied is a common water extraction herb; while the extraction process is originated from traditional Chinese medicine decoct processes. Traditional testing methods to verify the use of modern technology are reasonable, efficient, reliable and suitable for guiding modern industrial production processes.

\section{References}

[1] China Pharmacopoeia Committee (2010) Chinese Pharmacopoeia, Volume I, PRC. Chinese Medical Science and Technology Press, Beijing, 191.

[2] Zhang, H.Y. (2010) Clinical Application of Decoction of TCM Formula Granule. Journal of North China Coal Medical University, 12, 496-498. 